\title{
USERS' SATISFACTION WITH INTERNET SERVICE PERFORMANCE-BASED ON USER LOG
}

\author{
Member Ojebode $^{1}$ | Bernard Ijesunor Akhigbe*2
}

${ }^{1}$ Computer Science Department, University of Ibadan, Ibadan, Nigeria

${ }^{2}$ Department of Computer Science and Engineering, Obafemi Awolowo University, Ile-Ife, Nigeria

\section{Correspondence}

*Bernard Ijesunor Akhigbe, Department of Computer Science and Engineering, Obafemi Awolowo University, Ile-Ife, Nigeria. Email: biakhigbe@oauife.edu.ng

\section{Present Address}

Department of Computer Science and Engineering, Obafemi Awolowo University, Ile-Ife, 220282, Nigeria

\begin{abstract}
We are currently on the cusp of a digital era when people engage Internet services (Int-Sevs) ceaselessly for sundry purposes such as learning, teaching, and research. However, the lack of sufficient understanding of user satisfaction still poses a huge challenge to Int-Sevs adaption to users' dynamic needs and the provision of required services in real-time within the university's context. This understanding is needful concerning what influences the performance of the Int-Sevs of a university. This paper, therefore, analyses the user log of about $65000 \log$ items generated by 120 users of a university's internet services that were collected over three months. The mixed-method approach was adopted. Thus, the two-step clustering and crosstabulation techniques were applied to identify natural groupings and examine them for existing relationships (respectively) to determine the existence of user satisfaction. The results showed a significant association and relationship between user satisfaction and Key Performance Indicators (KPIs). The study concluded that with data and efficient techniques, KPIs with user-centric criteria like user satisfaction could be investigated to find what influences the performance of a university's IntSevs. The quality of users' experience was omitted and left to be considered in the future in a conceivably longitudinal study.
\end{abstract}

\section{KEYWORDS:}

Cross Tabulation, Internet Services, Two-step Clustering, User-Centricity and Satisfaction, Users' Log

\section{1 | INTRODUCTION}

The internet provides sundry services that are invaluable and, as such, have become an essential part of any university system. In modern society, where the university is a major player, the role of the internet has become overarching in everyday life of humans due to the Information and Communication (I\&C) services it offers. Universities the world over now inevitably and more than ever rely on the internet as a facility developed to provide satisfactory I\&C services ${ }^{[1]}$. As an essential facility in a 
university space, when the performance of the internet is satisfactory, users - stakeholders are encouraged to rely on its services continue to deliver on their mandate of teaching, research, learning, and performance of administrative duties ${ }^{[2]}$. All of these duties are possible because of the media ecosystem, which the internet digitally hosts. This also makes it possible to provide the services of social software provisioning, video sharing, and Web 2.0 applications that combine seamlessly to support the creation of user-generated content primarily. How students and academics learn, teach and do research and non-academics socialize and communicate have been inexorably impacted ${ }^{\sqrt{3}}$. The functionality of the Internet cognizance of the preceding should therefore be utmost and thus provide satisfactory services. In this paper's context, this is equivalent to Internet performance concerning the provision of Internet services in a university space. Unlike in the developed countries, university staff and students contribute a great deal financially to sustain the level of satisfactory services they get from the Internet facility available within their university. For instance, a monthly fee is deducted from staff salaries (academic and non-academic) who subscribe (though willingly) to an existing payment scheme where it exists. At the same time, students pay based on the duration of surfing they can afford per time. This way, staff, students, and the university administration work together to finance and sustain the Internet services they get from time to time. The culture is exclusive of government provision in terms of the maintenance of Internet infrastructure to better service delivery in return ${ }^{[4]}$.

The life-saving role of the internet has been highlighted aside from its usefulness in supporting sundry users' work ethics ${ }^{[5]}$. This makes it imperative to review its performance continually regarding the services it renders. The aim is to ascertain if the utility the services offer is satisfactory enough to be worth the financial sacrifices made by the trio of staff-student-university administration. What is paramount in this context is the realization of user satisfaction. User (or consumer) satisfaction is unavoidably dependent on the consumption of services. As such, it is a criterion measurable or evaluated using several factors 6 [ 7 and therefore used to understand the success of systems ${ }^{[8]}$. This can be in terms of Quality of Experiences (QoE) and Quality of Services (QoS) ${ }^{[0] 10]}$. Studies of this nature rely on users' data based on the usage of the services under investigation. These data are often from users' consumption of - in this case - Internet services that include their interactions and communications during the use of the internet at different times for sundry tasks (or events). Users' log contains this type of data from which information about Internet performance indicators can be found. The level of satisfaction or dissatisfaction of Internet services can be understood from the activities - tasks or events contained and recorded in users' log once the user login in. Onceinternet, loginsds of information are captured about the user's activities. These logs contain numerous types of events that represent representing experiences and behavior ${ }^{11}$. These events have been mined and analyzed from the Access Logs of Internet Users (ALoIU). They have resulted in useful information that has significantly improved system performance based on the knowledge gained [12] [13].

Users' logs are ALoIU and are also known as event log files or Internet access log files 13 .15. It has been relied on as a method to reveal the searching behavior of users ${ }^{[15]}$, learn about users' events ${ }^{[11]}$, discover patterns and the characteristics of Internet users' behavior after investigation ${ }^{[14}$, and so on. Some other information, which can be learned from users' logs, includes usage profiles, user engagements, knowledge of what is being searched, and the discovery of implicit linkages and usage patterns 14 [15]. However, to the best of our knowledge, the users' log of a university's Internet service has not been analyzed to identify the presence or absence of users' satisfaction based on Key Performance Indicators (KPIs). This paper seeks to bridge this gap by applying two-step clustering and cross-tabulation techniques to analyze the users' log of the University of Ibadan Internet services. The most related work to this is the study of Akanni et al. $\frac{16}{16}$ that assessed the operations of independent Internet network operators that operate within the university's space. Both the KPIs and the criterion of user satisfaction resulting from the study are contributions that other universities could use to determine the state of their Internet services' performance. The other contributions of the study include a user-centric methodology and information, which stakeholders can leverage to learn about what influences a university's Internet services from users' opinion. The study models can also be extended to investigate the performance of Internet services from other contexts aside from that of the university. The rest of the paper includes Sections on literature review and methodology in Sections 2 and 3, respectively. The study's result and discussion are found in Section 4, while its conclusion is in Section 5.

\section{2 | LITERATURE STUDY}

The first part of this section discusses the theoretical background that lies the foundation of this study. The second part of this section discusses previous work that related to this study. 


\section{1 | Theoretical Background}

In this paper's context, performance is theorized as both dependent on technology and the perception of users, particularly when system usage is involved. Therefore, a technology's service delivery and what users perceive of such delivery are conceptualized as key elements in measuring a system's performance. Measuring the performance of any system is necessary to guide improvement. This is because, from its results, several improvement options can be inferred. Like Lord Kelvin puts it, and as cited in Ferreira et al. 17], "what you cannot measure, you cannot improve." This is consistent with the century-old requirement to measure before improving. Based on this, system performance is characterized to support the practical and feasible dimensions of improvement. In the literature, performance measurement has been addressed mainly from the system/device-centric perspective ${ }^{[16}$. In contrast, a system's poor performance in service delivery can be investigated based on the tasks - activities carried out by users using the services. The lack of a sufficient body of theory to handle this highlights the plausibility of the contribution made in this paper. This contribution is based on the users of the university's Internet service to show how users perceive their use of existing Internet services. This perception was critical in the identification of KPIs to investigate users' satisfaction in this paper. The usage log of users has the opinion users hold that has been demonstrated by their use of the university's Internet service. In this paper's context, the underlying theoretical involved that underlies this is purely user-centric ${ }^{18}$. It underpins the identification of system performance from users' opinion as found in the log that contains the recorded events of users based on their use of (in this case) Internet services over some time. An identifiable challenge with this is the absence of measure(s) to investigate Internet service performance with User-satisfaction (Us-sat) as a criterion. This was addressed in this paper by identifying and conceptualizing KPIs from the log of users with cluster analysis.

Evaluative theories point to the center element of an evaluation process. For the User-centric Theory (UcT), its central element is the user. Hence, it supports user-oriented data by which certain decisions are made to measure performance [19]. This theory, therefore, presents the basis to use user-oriented data within the context of end-user computing measurement ${ }^{200}$. UcT becomes a fitting theory to underpin the evaluation of Internet service performance in this paper because of the need to assess the operational behavior of Internet services in terms of their service delivery potentials. However, operational behavior and service delivery potentials in the context of this paper can only be attested to by users. This made it necessary to employ user-centered data and Us-sat as a subjective criterion. Though this criterion tends to be ambiguous concerning conception, it can be doused. This paper, therefore, draws on the theory of user-centricity and pursues its attendant path of Positivism and Constructivism (Pos\&Cons). The motivation to pursue this path of Pos\&Cons comes from the need to justify using the qualitative part of the qualitative and quantitative research methodology in this paper. To this end, Pos\&Cons supported quantifiable data with qualitative variables from specific items ${ }^{[21}$. This implied that users' views were considered from the context of the university community. Thus users' opinions from their log based on the use of available Internet resource within the campus of a university was collected to carry out the research reported in this paper.

Us-sat's choice as a criterion was informed by the understanding that Us-sat depends on users' usage experiences, of which digital traces are available in the log files. As a user-centric criterion, Us-sat, which is also known as customer satisfaction, is thus conceptualized in the context of this paper as a user's feelings of pleasure or disappointment concerning the use of a service. The basis for this is a function of the outcome of users' identifiable comparison of how they perceive the performance of a service or product to their expectations ${ }^{6}$. This paper extended this conception to delineate the Us-sat as the criterion to meet this paper's goal to investigate the performance of a university's Internet service delivery system. However, the lack of literature on Us-sat as it pertains to this type of assessment highlights the importance of the contributions made in this paper. The scientific plausibility of the contribution(s) presented herein comes from two questions:

- Are there a convincing connection(s) between KPIs and the level of users' satisfaction with the services offered by the university's Internet service?

- What are the possible causes of the loss of Internet service(s) and eventual termination?

These questions, among others, guided the research, which results are reported in the paper. The questions seek to find the KPI, which would significantly influence the other KPIs and Us-sat. To avoid straying away from this paper's goal, efforts were made to exclude perspectives outside its scope. This includes the exclusion of some influential criteria on Us-sat such as the Quality of Internet Services (QoIS) from the perspective of User Experiences (UEs) and other essential criteria for measuring Internet services' quality that is in the entire signaling ladder diagrams ${ }^{[22}$. 


\section{2 | Related Works}

Internet performance has been measured mainly from the perspective of non-users. This effort, which has foot dragged, intends to investigate performance and unravel users' need(s) using suitable network measurement tools ${ }^{[23-25]}$. However, based on the literature, there is no known effort, which measures Internet performance based on its service delivery using users' satisfaction. In Nagaraja ${ }^{[26]}$, a systematic approach to improve and quantify Internet service availability was carried out. The cause of Internet service failure was established due to the Lack of Sufficient Knowledge (LoSK) about how the entire system operates [27]. Borzemski ${ }^{[27]}$ submitted that LoSK could make the investigation of Internet performance even difficult. It also shows that network problems, which are difficult to identify, do bother end-user usability issues and are part of what causes poor network performance. In a related and recent study, Polpinij and Name ${ }^{[28}$ developed a mining method to mine internet usage patterns of interesting inappropriate user behaviors. The scheme uses a generalized sequential pattern algorithm specializing in the sequential mining of patterns from real event logs. Remarkably results that showed an interesting increase and improvement in the QoS of internet service were achieved. Liston ${ }^{[29]}$ developed methods to gather data on Internet performance from several locations. The work highlighted the importance of measuring the performance of the internet. In Joumblatt et al. ${ }^{[30]}$, predictors were designed to study the causes of user dissatisfaction with the performance of applications used by users in network environments. Their research effort used quantization and feature selection to mitigate user feedback and poor performance episodes. The work, among other things, highlights user-oriented data for the study of user satisfaction.

Attempts have been made to deploy smart algorithms to assess how users perceive the QoS provided by Internet networks. Shaikh et al. ${ }^{\text {31] }}$ used criteria such as the control flags of TCP and requests from HTTP were used to examine users' behavior. Though abnormal behaviors were reported using the smart algorithm, the goal of monitoring user reaction(s) to network performance was realized to improve user experiences. The possibility of assessing the level of end-users irritation (i.e., frustration) of Internet use from performance data was highlighted in the literature 19 . The work showed significant implications on how assigned blame due to users' frustration when using the internet could be valid feedback that guides decision-making towards improving Internet service performance and the provision of content to end-users. Lin et al. ${ }^{32]}$ used a competitive-based scheme in the area of Internet performance measurement. The scheme successfully analyzed the relationship between the QoS and Quality of Experience (QoE) and showed how they improve network performance. In another research work, Chen and $\mathrm{Hu}{ }^{[3]}$ reported a survey report on methods and tools for traffic measurement and network performance, focusing on the protocol layer.

Similarly, the non-use or use of the services of the internet has been examined. In the literature, the provision of satisfactory Internet services was considered based on QoS and QoE ${ }^{[22] 32}$. In Haryadi ${ }^{[22]}$, the QoS and QoE metrics used the mean opinion score to assess issues that bother on providing satisfactory Internet services. Additionally, data rate was highlighted as a metric to assess the level of satisfaction Internet service providers make available to their customers. A periodical measurement test on the Internet network can be run to report its performance. This was highlighted by Bajpai and Schonwalder ${ }^{34}$ in a survey that resulted in a taxonomy of measurement platforms for Internet performance measurement. Scale, timeline, coverage, measurement tools, and architecture, among other features, were employed to describe the platform in detail.

In a much-related research work by Akanni et al. ${ }^{[16}$, the users' satisfaction with the Internet service providers in a university context was assessed. Download and upload activities were the variables applied to simply test the rate of data download and upload at different times using the RantCell App in an Infinix T627 smartphone. Interestingly, the research is quantitative, unlike this current one that used more variables and applied clustering and cross-tabulation technique. In other similar researches, the criterion that stirred the researchers' aim was simply the internet as a teaching tool and research asset ${ }^{[35}$, focusing squarely on the technical aspects of Internet performance ${ }^{36}$. The obvious contrasts of the research work reported in this paper and the related ones reviewed so far, which put this current work within the right and justifiable context to make its contribution within the end-user computing measurement literature, are the non-use of the (i) Us-sat criterion and (ii) log analytic method ${ }^{[12]}$. This gap highlights an important need in literature, which is the need for a criterion and methodology to assess Internet service delivery performance within the user and the context of the university.

\section{3 | MATERIAL AND METHOD}

The work reported in this paper used quantifiable data that are unstructured and from specific items that highlight users' opinions. These opinions are contained in the log of users and are representative of their activities. These activities are information from 
transactional data regarding the interactions between users and the University of Ibadan Internet; the case study used in this paper. The data were used anonymously to avoid any iota of sentiment attached following the practice in Sieverink ${ }^{[12]}$. One assumption made in this paper is the assumption that the data in the user log file represents the activities of users who must have used the Internet services of the case study university as many times as possible. The two-step cluster analytic technique 37 , 38, (see Algorithm 1) was used to identify available natural groupings.

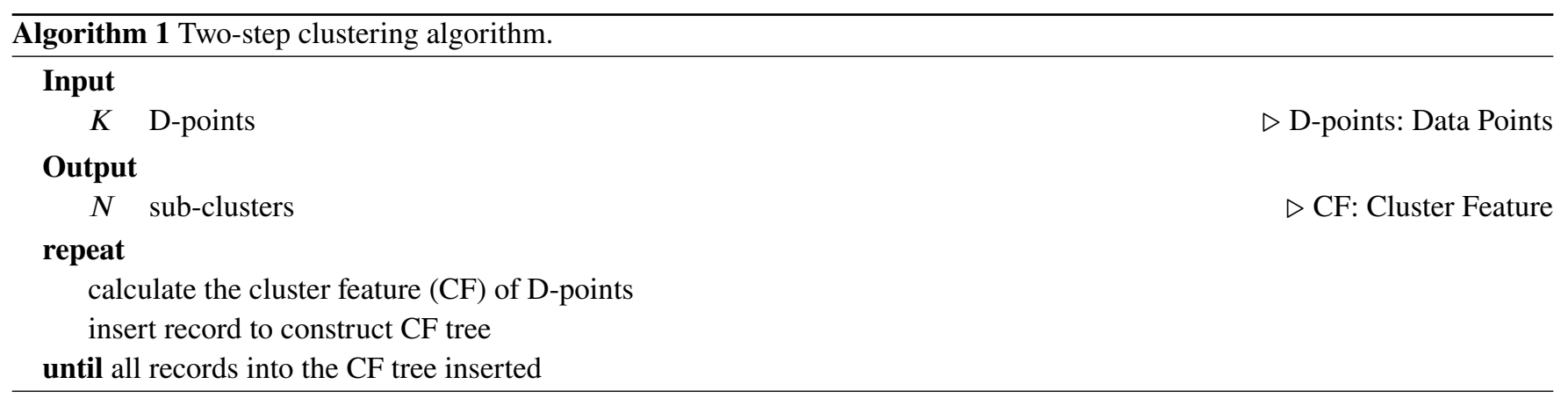

These natural groupings contain action patterns with users' behavior that depicts user-satisfaction, which are learned from within the dataset in the user log file. Its suitability informed the procedure's choice in Algorithm 1 for the categorical data type in the user $\log$ files. More details about the procedure can be found from Şchiopu ${ }^{\text {[39] }}$ and Tang et al. ${ }^{\text {38. }}$. The likelihood distance measure was applied to bring about the continuous use of categorical data so that both normal (Gaussian) and multinomial distribution were not missing ${ }^{400}$. This concise representation of the relationships (patterns) entrenched in the data was subjected to correlation analysis. By this, the strength and direction of the relationship that exists among natural groupings were identified. The cross-tabulation technique ${ }^{41]}$ (see Algorithm 2) was then applied to examine these relationships (cell contents) to establish independence, measures of association, and agreement.

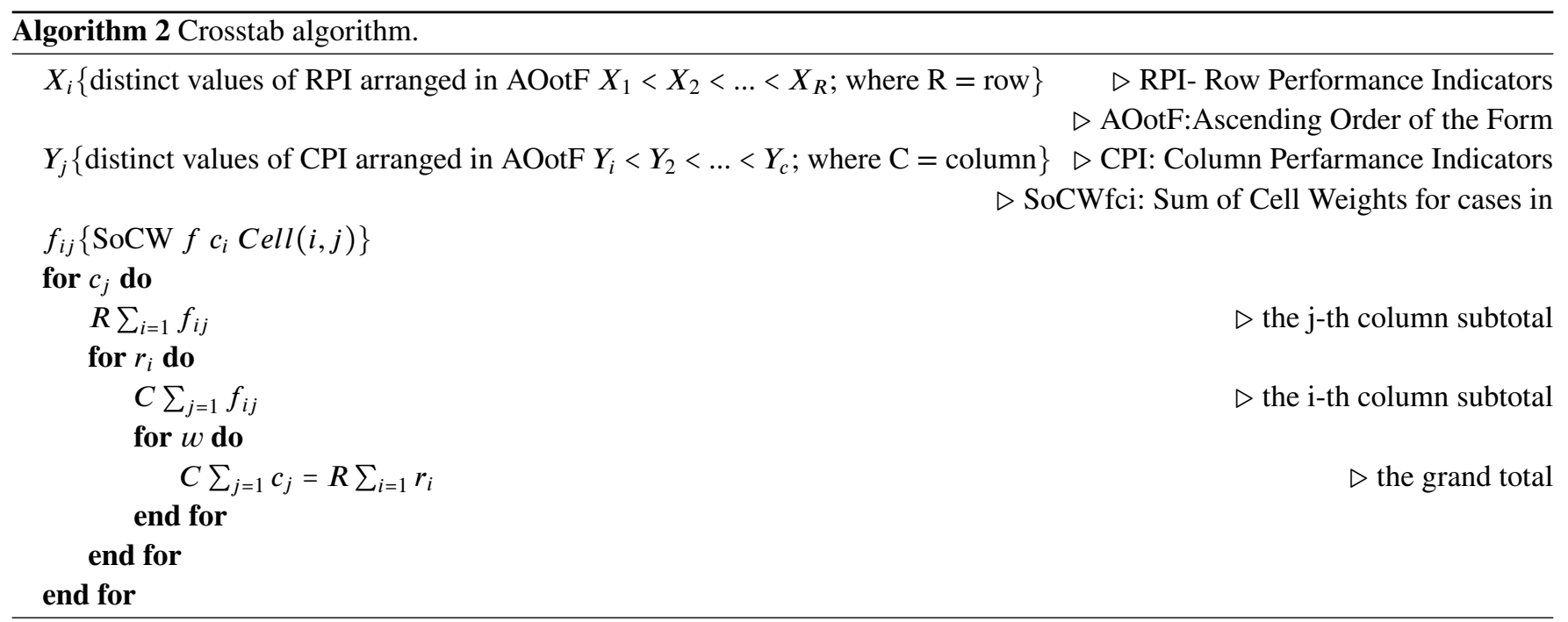

The cross-tabulation technique used the performance indicators to examine the clusters found to ascertain if they provided and possess an adequate level of user satisfaction. By this, each cluster was tested to determine the performance indicator with the most significant influence on Internet performance. To further process the log files' information, the performance dimension was reconciled and adopted as Performance indicators that were initially referred to as users' log containing variables. This formalism helped further process data processing in the log file to discover more information. To this end, four KPIs (Distance, Session Time, Upload and Download) were therefore put forward as input variables and three (NASIP Address - Network Access Server 
Internet Protocols Address, network, and causes of termination) evaluators variables to analyze the level of users' satisfaction from users' log items - data. About 65,000 log items, which more than 120 users generated, were collected within three months following best practice as demonstrated in Tao et al. ${ }^{15 .}$. These log items are the activities of users. The IBM SPSS 20 was used to implement two-step cluster analytic and the crosstab technique. Since two main types of networks - fiber and wireless were observed to provide Internet services to users within the university community, download data was binned (i.e., grouped) for analysis to avoid bias. This motivated the use of cross-classification of data with a cross-tabulation technique to determine whether users used the wireless and/or fiber-optic network for their download activities. The belief is that this will give a better rating of users that reflects the actual quality of download at each cluster.

\section{4 | RESULTS AND DISCUSSION}

This section is devided into three parts. The first part presents the analysis result of two step-cluster. The second part presents the result of cross-tabulation. The last part discusses those two results.

\section{1 | Two Step-Cluster Analysis Result}

The four variables that served as input variables - Upload, Download, Session Time, and Distance were used to create clusters. Two variables were used as evaluation variables, namely NASIP Address, network, and causes of termination. Algorithms 1 and 2 are the procedures IBM SPSS 20 applied to identify the natural groupings and examine the relationships (cell content) in the groupings, respectively. The cluster analysis result based on input and evaluation variables is shown in Figure 1 using the model summary of IBM SPSS 20. Four variables (e.g. Upload - or AcctInputOctets, Download - AcctOutputOctets, Session Time, and Distance, formed the models presented after clustering. Analysis results of these variables are shown in the input (predictor) section in Figure 1. But as a matter of importance, it is needful to note that the color of the variables is the saw with the color inside the red rectangular broken line in Figure 1. The predictor importance also revealed that the sum of all predictors is 1.0, as depicted by the color. The information in Figure 1 has a striking implication. This is because the result from Figure 1 based on cluster information implies more download - AcctOutputOctets (i.e., response) with the tendency for users to carry out upload - AcctInputOctets activities. This means that users' activities on the internet increased.

The value - 1.0 on the top right-hand side of the diagram in Figure 1 and within the highlighted red rectangular broken line affirms that the model built using the clustering technique is a "good Model." This value reveals the Input (Predictor) Importance of the model showing in Figure 1. It highlights the Input and Evaluation fields' plausibility under the description section in the first column on the diagram's left-hand side. The values also indicate that the silhouette value is way above +5 , thus affirming the model's validity built from the clustering analysis. From the model in Figure 1, the cluster sizes show the Sizes of the Clusters in Percentage (\%) (SoCiP) and the Volume (amount) of Users' Activities (VoUA) in numerals. For example, the model in Figure 1 reveals the cluster sizes in (\%) of clusters 1, 2, 3, and 4 to be $37.7 \%, 4.3 \%, 28.4 \%$, and 29.6\%; and VoUA as 23169, 2647, 17423, and 18211 respectively. Both values of clusters in SoCiP and VoUA represent the log item records in each cluster (see the size row in Figure 1).

Empirically, cluster 1 with $37.7 \%$ (i.e., 23,169 users' activities) shows that the lowest and highest data use account is 4,248,016.92 and 46,897,975.46 in terms of upload and download, respectively. Interestingly, a total of 3,246.47 session time (AcctSessionTime) was expended when the network (uptime) was delivering Internet services up to 100\% (see Evaluation Field in Figure). It follows that as the network's performance dwindles from $100 \%$ to $72.9 \%, 56.6 \%$ and $56.3 \%$ more session time (e.g., 18,315.78) was incurred at the network (uptime) of $72.9 \%$ since users were still optimistic that the network would improve. But as it dwindled, the session time began to drop. From the evaluation field; the influence of the variable - Lost of Service (L-S) that refers to the cause of the Termination of Internet Connection (AcctTerminateCause) on variables in the input field shows that as the network (uptime) dwindle, L-S also wane in the same proportion across all the clusters.

Cell Distribution is made up of clusters. In this paper, four clusters were identified, and the Cell Distribution Results (CDRs) in Figure 2 are for only Distance. However, for brevity, the CDRs of the remaining three input variables were not presented. CDR was used to specify the frequency of count and the distribution of Users' Activities (UssAct). The count represents the number of users linked with the UssAct. For the Distance input variable, which CDR is presented in Figure 2, cluster 1 only shows a concentration of UssAct in distances 4 and 5. In cluster 2, UssAct were observed in distances 1 through 5. The fuzzy explanation 


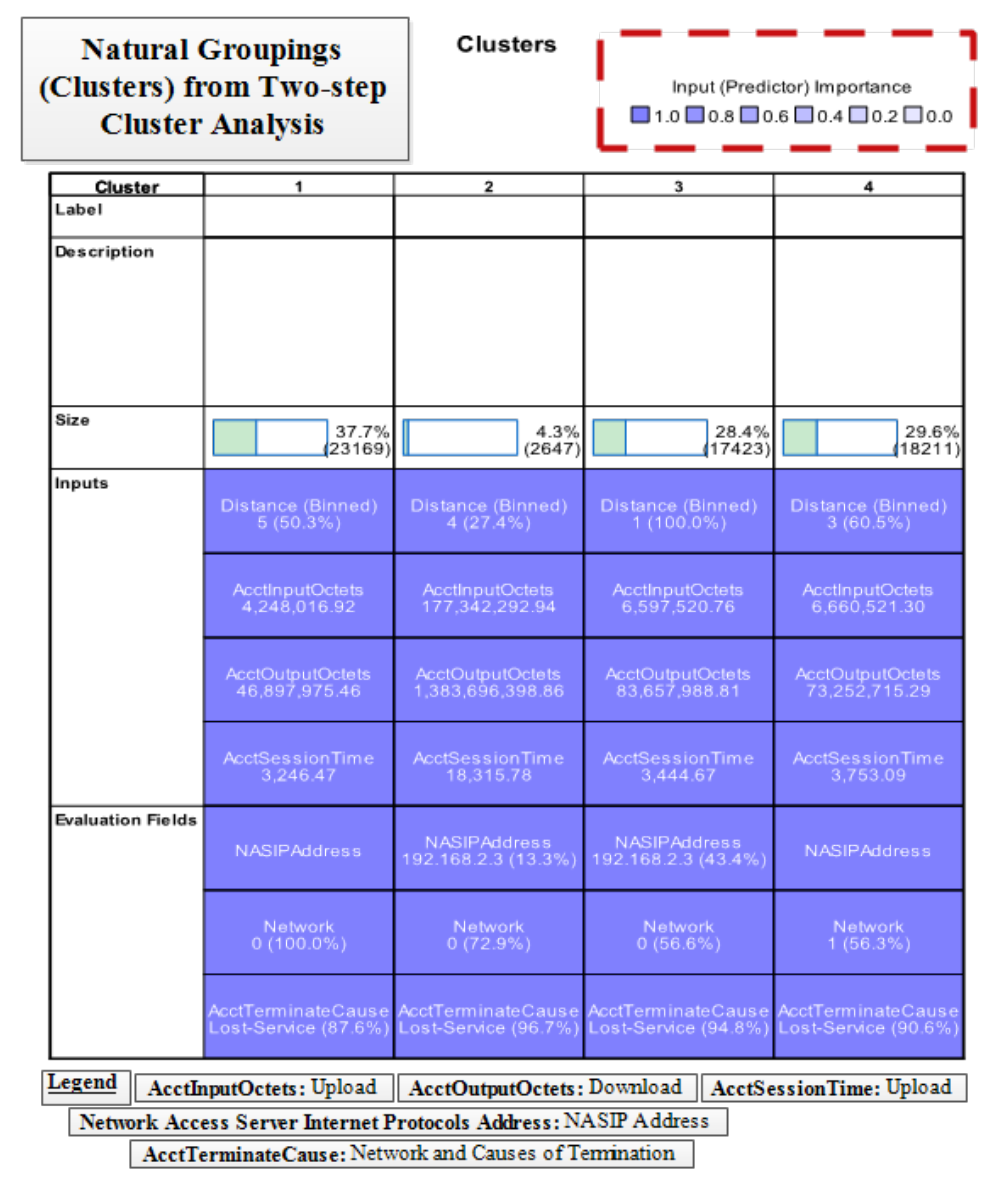

FIGURE 1 Information about clusters.

TABLE 1 Chi-square result showing strong evidence of a relationship.

\begin{tabular}{lllc}
\hline & Value & df & $\begin{array}{c}\text { Asymp. Sig. } \\
\text { (2-sided) }\end{array}$ \\
\hline Pearson Chi-Square & $8381.603 \mathrm{a}$ & 9 & .000 \\
Likelihood Ratio & 6978.152 & 9 & .000 \\
Linear-by-Linear Association & 416.789 & 1 & .000 \\
N of Valid Cases & 61450 & & \\
\hline $\begin{array}{l}\text { a. 0 cells (0.0\%) have an expected count less than 5. } \\
\text { The minimum expected count is 518.46; and significant level }(\alpha)=0.05 .\end{array}$
\end{tabular}

for this is that the NAS may have sustained the Internet services' uptime in all the distances. This, however, is still subject to further investigation, which is left for another study. It can also be observed that the number of users linked with UssAct was at an all-time low, with less than 2000 users linked to the UssAct generally in cluster 2 . The act in cluster 3 was only observed in distance 1 , while in cluster 4 , the act was noticed in distances 2 and 3. The size of the clusters in terms of SoCiP is indicative of the volume of UssAct that took place cognizance of the total (100\%) volume of data under analysis.

\section{2 | Cross-Tabulation Result}

The result from cross-tabulation analysis is presented using Chi-square following Wendler and Grottrup ${ }^{41}$. This result is presented in Table 1. Usually, the likelihood ratio results and linear-by-linear association are ignored since they are most suitable for quantitative variables, unlike the qualitative variables used in this study. Therefore, the Pearson Chi-Square result is upheld since it is satisfactory to test for a statistically significant relationship between qualitative variables. 


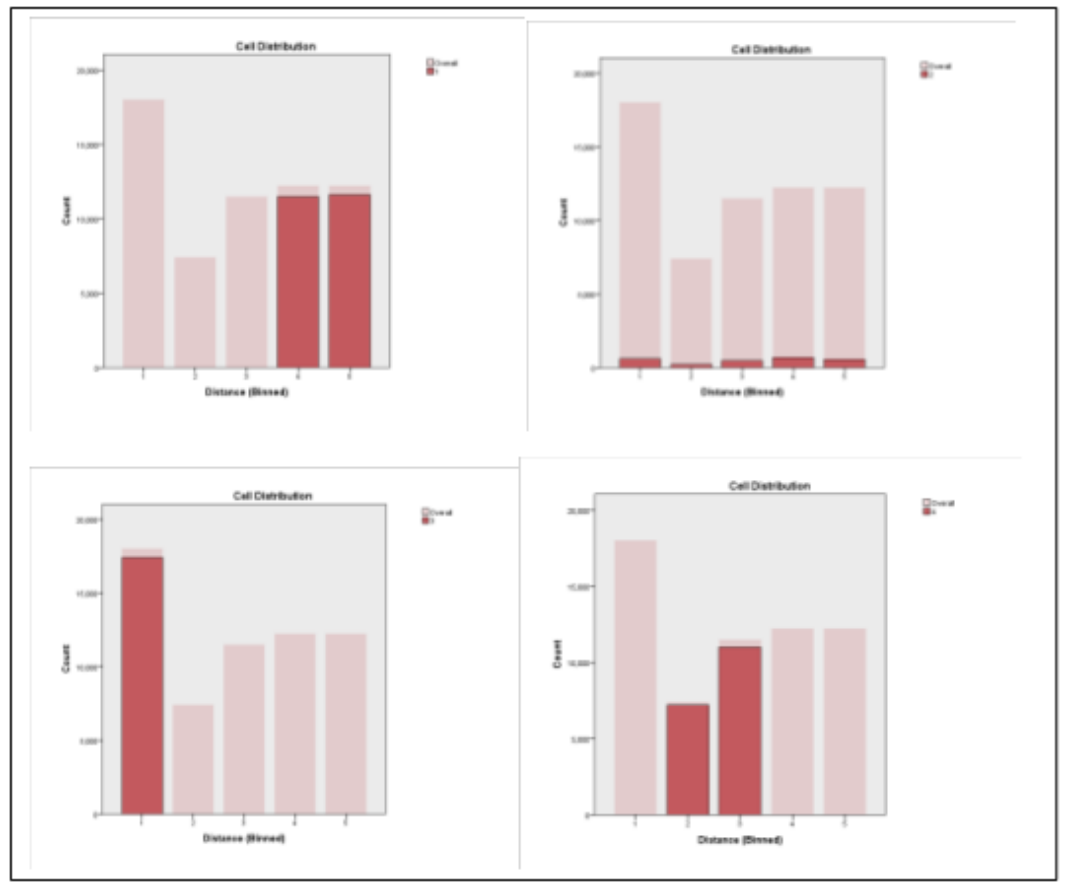

FIGURE 2 Cell distribution of distance variable showing the number of users' activities.

TABLE 2 Correlations matrix showing the relationship between distance, session time, upload, and download.

\begin{tabular}{|c|c|c|c|c|}
\hline Var./kPIs & Dist & Ses-Time & Upload & Download \\
\hline Dist. & 1 & & & \\
\hline Ses-Time & .003 & 1 & & \\
\hline Upload & .000 & $.221 * *$ & 1 & \\
\hline DwnLd & $-.021 * *$ & $.427 * *$ & $.381 * *$ & 1 \\
\hline
\end{tabular}

In the result of Pearson Chi-Square in Table 1, the p-value is $=(p<0.000)$ and the significant level $(\alpha)=0.05$. Therefore, it is concluded that there is very strong evidence of a relationship between user satisfaction and KPIs due to the influence of evaluator variables based on the results of (Chi-Square Tests $=8381.603$, confidence interval $(\mathrm{df})=9$, and $\mathrm{p}<0.000$ ). The study also resulted in some other interesting results, which depict relationships between the KPIs that were inputted for analysis. These results were captured using a Correlation Matrix that showed very stimulating results concerning the relationship between the four input KPIs, namely Distance, Session Time, Upload, and Download indicators (see Table 2).

The results presented in Table 2 are based on a 2-tailed statistical analysis, in which correlation is significant at the 0.01 level. This implies that the results of interest are the results that indicate the existence of the relationship between Upload and Session Time $\left(0.221^{* *}\right)$; Download and Distance (-0.021**); Download and Session Time (0.427**); and Download and Upload (0.381**). These relationships are based on their correlation, which is significant at the 0.01 level. By implication, the relationship between Upload and Session Time $\left(0.221^{* *}\right)$ shows that session time impacts download. This implies that Download activities were prevalent whenever there were session times. The results also show that Distance was correlated with download (-.021), thus informing that there were more download activities within the Internet Access Nodes (IAN) closest to the NAS - Network Access Server. This also implies that the closer the IAN is to the NAS, the more and better the download services users get. Form both relationships (e.g., Upload and Session Time (0.221**) and Distance and download (-.021)); it was observed that users spend a lot of time using the Internet services when it was possible to carry out more upload and download activities. This implied that the more time users could spend on the internet, given that the condition of Session Time and Distance are met, the more satisfied they were. Furthermore, the correlations between Download and Session Time (0.427**) and Download and Upload $\left(0.381^{* *}\right)$ indicators also show that when there was session time, both download and upload activities were visible. Similarly, 
TABLE 3 Shows the ISDE of users in the four clusters based on Distance.

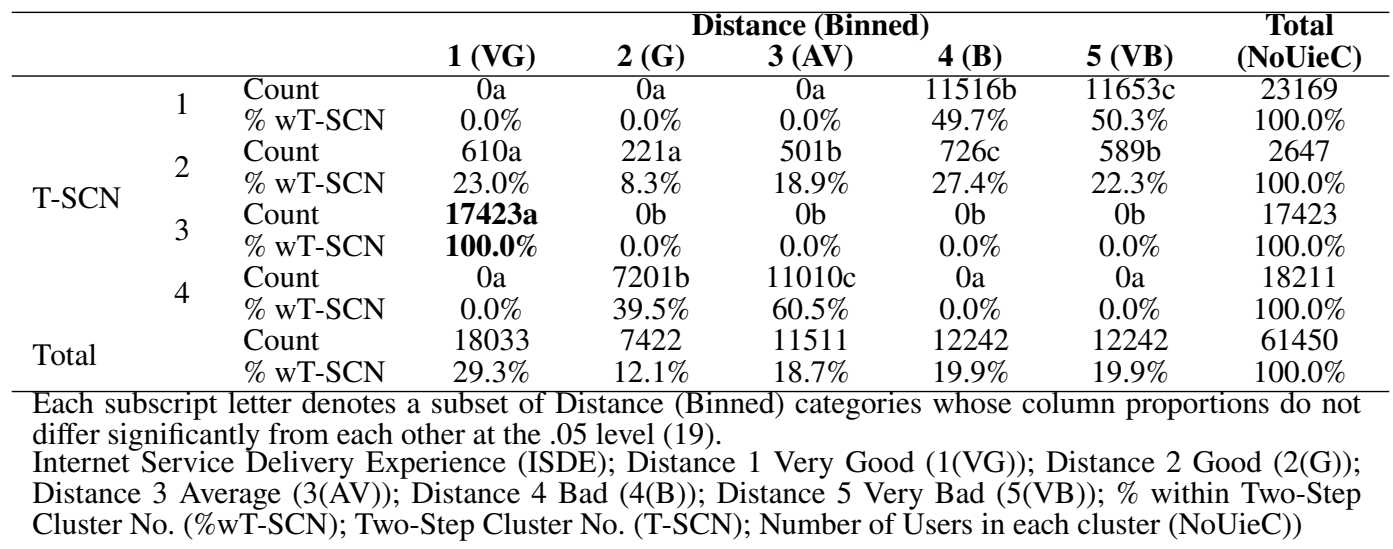

TABLE 4 Shows the ISDE of users in the four clusters based on upload.

\begin{tabular}{|c|c|c|c|c|c|c|c|c|}
\hline & \multicolumn{5}{|c|}{ Upload (Binned) } & \multirow{2}{*}{$\begin{array}{c}\text { Total } \\
\text { (NoUieC) }\end{array}$} \\
\hline & & & $\begin{array}{c}\leq \mathbf{2 2 3 7 8 6} \\
\text { (VG) }\end{array}$ & $\begin{array}{c}\leq 1025370 \\
(G)\end{array}$ & $\begin{array}{c}\leq 2670096 \\
(\mathrm{AV})\end{array}$ & $\begin{array}{c}\leq 7202424 \\
\text { (B) }\end{array}$ & $\begin{array}{l}\geq 7202425 \\
\text { (VB) }\end{array}$ & \\
\hline \multirow{8}{*}{ T-SCN } & \multirow{2}{*}{1} & Count & $6091 \mathrm{a}$ & $5039 b$ & $4647 \mathrm{c}$ & $4282 d$ & $3110 \mathrm{e}$ & 23169 \\
\hline & & $\%$ wT-SCN & $26.3 \%$ & $21.7 \%$ & $20.1 \%$ & $18.5 \%$ & $13.4 \%$ & $100.0 \%$ \\
\hline & \multirow{2}{*}{2} & Count & $14 \mathrm{a}$ & $15 a$ & $44 b$ & $172 \mathrm{c}$ & 2402d & 2647 \\
\hline & & $\%$ wT-SCN & $0.5 \%$ & $0.6 \%$ & $1.7 \%$ & $6.5 \%$ & $90.7 \%$ & $100.0 \%$ \\
\hline & \multirow{2}{*}{3} & Count & $2479 a$ & $3602 b, c$ & $3769 c$ & $4117 d$ & $3456 b$ & 17423 \\
\hline & & $\%$ wT-SCN & $14.2 \%$ & $20.7 \%$ & $21.6 \%$ & $23.6 \%$ & $19.8 \%$ & $100.0 \%$ \\
\hline & \multirow{2}{*}{4} & Count & $3457 \mathrm{a}, \mathrm{b}$ & $3659 \mathrm{~b}, \mathrm{c}$ & $3897 d$ & $3857 \mathrm{c}, \mathrm{d}$ & 3341a & 18211 \\
\hline & & $\%$ wT-SCN & $19.0 \%$ & $20.1 \%$ & $21.4 \%$ & $21.2 \%$ & $18.3 \%$ & $100.0 \%$ \\
\hline \multirow{2}{*}{ Total } & & Count & 12041 & 12315 & 12357 & 12428 & 12309 & 61450 \\
\hline & & $\%$ wT-SCN & $19.6 \%$ & $20.0 \%$ & $20.1 \%$ & $20.2 \%$ & $20.0 \%$ & $100.0 \%$ \\
\hline
\end{tabular}

The subscript-letter in this table represents a subset of Upload (Binned/per unit). The column proportion(s) is not significantly different from others at the .005 level.

Internet Service Delivery Experience (ISDE); Satisfied (S); Very Dissatisfied (VDS); Dissatisfied (DS);

Very Satisfied (VS); \% within Two-Step Cluster No. (\%wT-SCN); Two-Step Cluster No (T-SCN)

users who were able to carry out download activities when necessary were also able to carry out upload activities and vice versa successfully.

Conclusively, both Distance and Session Time variables are factors to harness when contemplating the provision or improvement of Internet Connectivity and Service Delivery (IC\&SD) in the University space. If the provision of IC\&SD is particularly intended to cover a large expanse of land, it is inevitable to use the right technology for optimum performance. This summation is consistent with the provisions in Berba and Palaoag ${ }^{42]}$ and Munyanti and Mason 4 in terms of upload and download activities as factors to improve user satisfaction of Internet use. It also agrees with Ghose and Han's research effort 44 , who concluded that the extent of geographical mobility of users (Distance) has a positive effect on Internet activities. The cross-tabulation technique also gave some other interesting results aside from the Chi-square and Matrix Correlation results shown in Tables 1 and 2. The results were meant to show the statistical significance level in the relationship between input variables versus that of the evaluator variables and user satisfaction. The results in Tables 3, 4, 5, 6, 7, and 8, which are also from the crosstabulation analysis, are meant to show further that the qualitative variables depend on each other with sufficient statistically significant relationships conclude from the results in Tables 1 and 2 . These dependencies are additional proof of the existence of a relationship between user satisfaction and KPIs. It is interesting to note that only cross-tabulation results are presented for Distance, Upload, Download, and Download experiences for both wireless (mobile) and fiber-based network for brevity. The results were binned (i.e., values were grouped into the same category to maximize interpretation). In Table 3 , the results are grouped based on categories from Very Good (VG) to Very Bad (VB). In this study, the Distance 1(VG), 2(G), 3(AV), 4(B), and $5(\mathrm{VB})$ means the Distance of users' Internet Access Node (IAN) that is closest, closer, close, far and farther away (respectively) from the ICT center where the NAS is located. 
TABLE 5 Shows the ISDE of users in the four clusters based on the download.

\begin{tabular}{|c|c|c|c|c|c|c|c|}
\hline & & & & Downloa & (Binned) & & Total \\
\hline & & & $\begin{array}{c}\leq 1125352 \\
(\text { VDS })\end{array}$ & $\begin{array}{c}\leq 6843847 \\
(\mathrm{DS})\end{array}$ & $\begin{array}{c}\leq 89746116 \\
\text { (S) }\end{array}$ & $\begin{array}{c}\geq 89746117 \\
\text { (VS) }\end{array}$ & Total \\
\hline & & Count & $6058 \mathrm{a}$ & $5130 b$ & $8910 \mathrm{c}$ & $3071 d$ & 23169 \\
\hline & 1 & $\%$ wT-SCN & $26.1 \%$ & $22.1 \%$ & $38.5 \%$ & $13.3 \%$ & $100.0 \%$ \\
\hline & & Count & $15 a$ & 23a & $390 b$ & $2219 c$ & 2647 \\
\hline T SCN & 2 & $\%$ wT-SCN & $0.6 \%$ & $0.9 \%$ & $14.7 \%$ & $83.8 \%$ & $100.0 \%$ \\
\hline $1-S C N$ & & Count & $2396 a$ & $3415 b$ & $7776 c$ & $3836 \mathrm{c}$ & 17423 \\
\hline & 3 & $\%$ wT-SCN & $13.8 \%$ & $19.6 \%$ & $44.6 \%$ & $22.0 \%$ & $100.0 \%$ \\
\hline & 4 & Count & $3567 a$ & $3744 a, b$ & $7702 b$ & $3198 c$ & 18211 \\
\hline & 4 & $\%$ wT-SCN & $19.6 \%$ & $20.6 \%$ & $42.3 \%$ & $17.6 \%$ & $100.0 \%$ \\
\hline & & Count & 12036 & 12312 & 24778 & 12324 & 61450 \\
\hline Total & & \%wT-SCN & $19.6 \%$ & $20.0 \%$ & $40.3 \%$ & $20.1 \%$ & $100.0 \%$ \\
\hline
\end{tabular}

The proportions of a column of the categories of download (Binned) do not significantly differ from each other at the 0.05 level and are denoted by each letter's subscript. \% within Two-Step Cluster No. Internet Service Delivery Experience (ISDE); Satisfied (S); Very Dissatisfied (VDS); Dissatisfied (DS); Very Satisfied (VS); (\%wT-SCN); Two-Step Cluster No (T-SCN)

TABLE 6 Shows the ISDE of users in the four clusters based on download and Network Types.

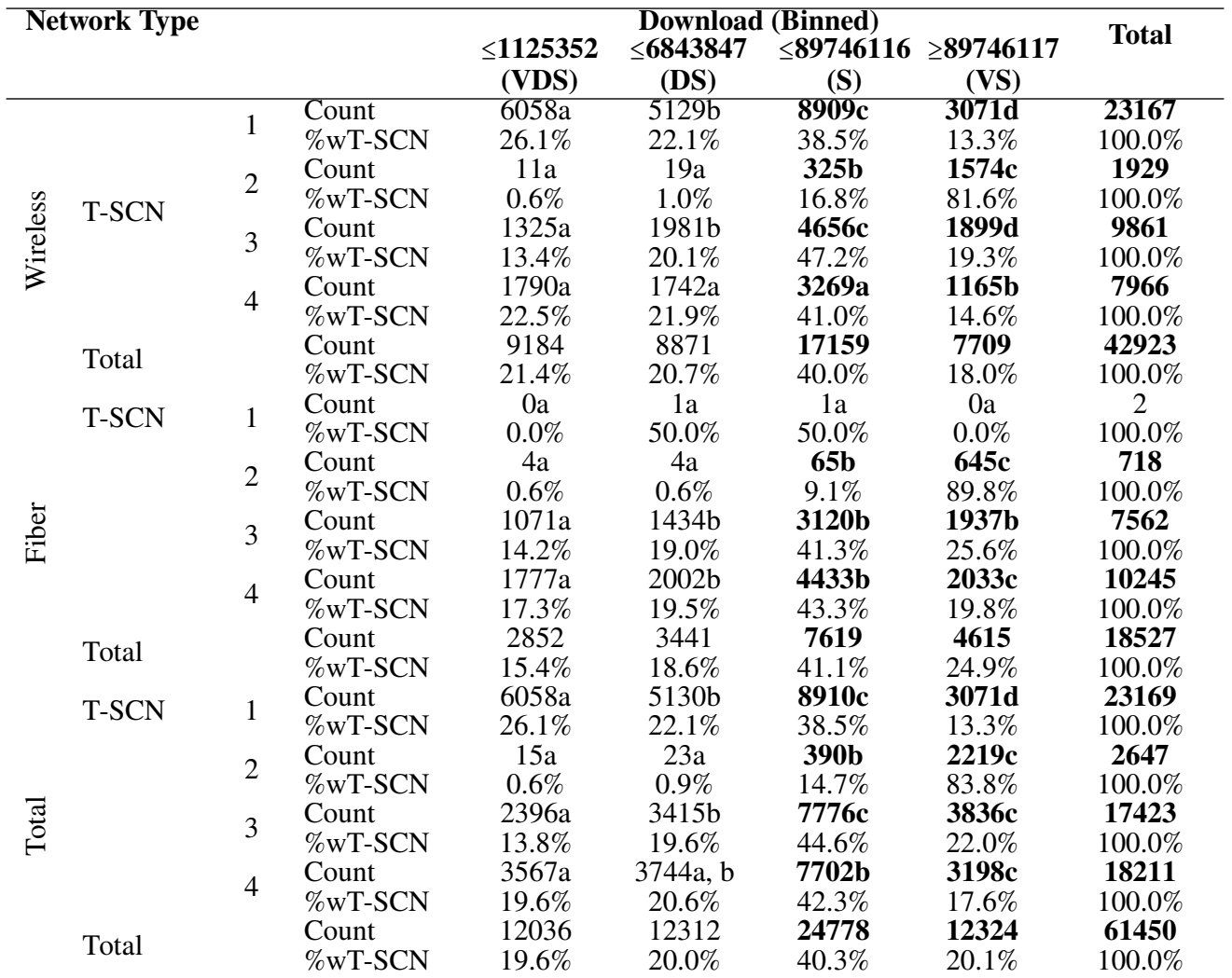

Each subscript letter denotes a subset of Download (Binned) categories whose column proportions do not differ significantly from each other at the .05 level. Internet Service Delivery Experience (ISDE); Very Dissatisfied (VDS); Dissatisfied (DS); Satisfied (S); Very Satisfied (VS); \% within Two-Step Cluster No. (\%wT-SCN); Two-Step Cluster No (T-SCN)

Table 3 shows the number of user activities in each cluster based on the impact of the Distance variable on Internet service delivery performance. In cluster 3, though users' location was within a very good distance, the performance of the internet was still poor (See Highlighted Value in bold (SHViR)). It was found that the population of users in the cluster was too high and, as such, overwhelmed the network strength ${ }^{[16]}$. From the results in Table 4, it is evident that the number of users within the VG - very good category of upload is very low in all the clusters (except in cluster 2) when compared to the population of users within each cluster. Averagely, the distribution of users across the available range of upload categories - VB, B, AV, G, and 
TABLE 7 Shows the ISDE of users in the four clusters based on session time.

\begin{tabular}{|c|c|c|c|c|c|c|c|c|}
\hline & & & \multicolumn{5}{|c|}{ Session Time (Binned) } & \multirow{2}{*}{ Total } \\
\hline & & & $\begin{array}{l}\leq 851 \\
(\mathrm{VG})\end{array}$ & $\begin{array}{c}\leq 1931 \\
(G)\end{array}$ & $\begin{array}{c}\leq \mathbf{3 5 0 5} \\
(\mathrm{AV})\end{array}$ & $\begin{array}{c}\leq \mathbf{6 2 9 3} \\
\text { (B) }\end{array}$ & $\begin{array}{c}\geq 6294 \\
(\mathrm{VB})\end{array}$ & \\
\hline \multirow{8}{*}{ T-SCN } & \multirow{2}{*}{1} & Count & $5404 a$ & $5006 b$ & $4738 \mathrm{c}$ & $4518 \mathrm{c}$ & $3503 \mathrm{~d}$ & 23169 \\
\hline & & $\%$ wT-SCN & $23.3 \%$ & $21.6 \%$ & $20.4 \%$ & $19.5 \%$ & $15.1 \%$ & $100.0 \%$ \\
\hline & \multirow{2}{*}{2} & Count & $0 \mathrm{a}$ & $3 a$ & $31 b$ & $218 \mathrm{c}$ & $2395 d$ & 2647 \\
\hline & & $\%$ wT-SCN & $0.0 \%$ & $0.1 \%$ & $1.2 \%$ & $8.2 \%$ & $90.5 \%$ & $100.0 \%$ \\
\hline & \multirow{2}{*}{3} & Count & $3652 \mathrm{a}$ & $3644 a$ & $3733 a$ & $3615 \mathrm{a}$ & $2779 b$ & 17423 \\
\hline & & $\%$ wT-SCN & $21.0 \%$ & $20.9 \%$ & $21.4 \%$ & $20.7 \%$ & $16.0 \%$ & $100.0 \%$ \\
\hline & \multirow{2}{*}{4} & Count & $3306 a$ & $3685 b$ & $3856 b, c$ & $3985 c$ & $3379 a$ & 18211 \\
\hline & & $\%$ wT-SCN & $18.2 \%$ & $20.2 \%$ & $21.2 \%$ & $21.9 \%$ & $18.6 \%$ & $100.0 \%$ \\
\hline \multirow{2}{*}{ Total } & & Count & 12362 & 12338 & 12358 & 12336 & 12056 & 61450 \\
\hline & & $\%$ wT-SCN & $20.1 \%$ & $20.1 \%$ & $20.1 \%$ & $20.1 \%$ & $19.6 \%$ & $100.0 \%$ \\
\hline
\end{tabular}

Each subscript letter denotes a subset of Session Time (Binned) categories whose column proportions do not differ significantly from each other at the .05 level.

Internet Service Delivery Experience (ISDE); Satisfied (S); Very Dissatisfied (VDS); Dissatisfied (DS);

Very Satisfied (VS); \% within Two-Step Cluster No. (\%wT-SCN); Two-Step Cluster No (T-SCN)

VG was observed for each binned upload to be approximately $20 \%$ (SHViR). Thus, in clusters 1, 3, and 4, the spread of users across the binned upload was observed to be substantial, unlike in cluster 2, where fewer users' activities were seen with a high concentration around the VG - very good upload category. Table 5 shows an equivalent level of users' satisfaction with Internet service delivery based on download activities. This is because $40.3 \%$ of users' activities were observed to fall within the satisfied (S) category compared to other categories. It was also obvious that Vusers' distribution was on average approximately $20 \%$ each (see Table 5). This result also exemplifies the spread of users across clusters. For cluster 2, where fewer users' were noticed, a slight and continuous increase from VDS, DS, and S to VS categories was noticed. The VS category in cluster 2 with 2219 users' activities demonstrates that users would ordinarily gravitate towards where they can get better download services.

Results in Table 6 show users Internet Service Delivery Experiences (ISDE) with the wireless or fiber-optic network. With further cross-classification, the result of the total distribution of users among the binned download for wireless presents the values of VDS, DS, S, and VS as $21.4 \%, 20.7 \%, 40.0 \%$, and $18.0 \%$, respectively. These results showed interesting contrasts with binned fiber optics, with VDS, DS, S, and VS having 15.4\%, 18.6\%, 41.1\%, and 24.9\%, respectively. This implies that users of the services were more satisfied with the wireless network's services than the one provided by the wired fiber-optic connection.

It was observed from the results in Table 6 that users' activities were seen to concentrate more at the Satisfactory and Very Satisfactory categories for both the wireless and fiber network means of Internet service delivery. The results between fiber and wireless type of download activities showed that network type could also potentially impact significantly on users' perception of satisfactory Internet performance concerning service delivery. For example, Cluster 1 shows poor performance with just two users on the fiber-optic Internet network, while cluster 2 showed the best performance and had 645 users' activities. Clusters 3 (with 10245 users' activities) and 4 (with 18527 users' activities) with more users on the fiber optic still recorded poor performance. This shows that a Network's strength should be able to support the number of users relying on it pert time. This means that users' population can affect a network irrespective of fiber optic or wireless network type, which affirms the claim in Akanni et al. 16 .

Table 7 is based on the influence of session time based on users' Internet Service Delivery Experience (ISDE). At each binned session time, the relative distribution of users seeking ISDE was observed. The approximate average of $20 \%$ spread of users across the session time binning highlights this. This spread is peculiar to clusters 1,3 , and 4 , with a sharp contrast of users spread across the binned session time in cluster 2 with $90.5 \%$ of very good session time. This result also shows that users, in their bid to get satisfactory ISDE, would normally settle for where the session time is better as observed with download activities.

In Table 8, the result showed the Lost of Service (L-S) indicator to be a major reason why users ISDE were often terminated. The recordings about the terminate cause account across the nodes (i.e., IAN) and within all the clusters are a testament to this. Among other factors (see Table 8), L-S showed the strongest influence as the cause of Internet service termination. The record showed that 55851 users $(90.9 \%)$ out of the 61450 activities of users (100\%) reflected the finding. While L-S had $90.9 \%$ influence, the other factors barely trailed this huge influence with a total of $9.1 \%$. This result was further confirmed in Cluster 2 with more concentration of users' activities, up to $96.7 \%$ found under L-S. 
TABLE 8 Shows the ISDE of users in the four clusters based on the cause of service termination.

\begin{tabular}{|c|c|c|c|c|c|c|c|c|c|c|}
\hline & & & & & Ses & n Time ( & ned) & & & Total \\
\hline & & & $\begin{array}{l}\text { Other } \\
\text { Causes }\end{array}$ & $\begin{array}{l}\text { Admin } \\
\text { Reboot }\end{array}$ & $\begin{array}{c}\text { Admin } \\
\text { Reset }\end{array}$ & $\begin{array}{c}\text { Lost } \\
\text { Carrier }\end{array}$ & $\begin{array}{l}\text { Lost of } \\
\text { Service }\end{array}$ & $\begin{array}{c}\text { Session } \\
\text { Timeout }\end{array}$ & $\begin{array}{c}\text { User } \\
\text { Request }\end{array}$ & lotal \\
\hline & & Count & $2176 a$ & $0 \mathrm{~b}, \mathrm{c}, \mathrm{d}, \mathrm{e}$ & $4 e$ & $150 \mathrm{a}$ & 20290d & $0 \mathrm{c}, \mathrm{e}$ & $549 b$ & 23169 \\
\hline & 1 & $\% \mathrm{wT}-\mathrm{SCN}$ & $9.4 \%$ & $0.0 \%$ & $0.0 \%$ & $0.6 \%$ & $87.6 \%$ & $0.0 \%$ & $2.4 \%$ & $100.0 \%$ \\
\hline & 2 & Count & $13 a$ & $0 \mathrm{a}, \mathrm{b}$ & $4 b$ & $6 \mathrm{~b}$ & $2559 b$ & $0 \mathrm{a}, \mathrm{b}$ & $65 b$ & 2647 \\
\hline & 2 & $\%$ wT-SCN & $0.5 \%$ & $0.0 \%$ & $0.2 \%$ & $0.2 \%$ & $96.7 \%$ & $0.0 \%$ & $2.5 \%$ & $100.0 \%$ \\
\hline I-SCN & & Count & $144 a$ & $12 b$ & $30 \mathrm{c}$ & $63 d$ & 16510d & $0 \mathrm{a}$ & $664 c$ & 17423 \\
\hline & 3 & $\% \mathrm{wT}-\mathrm{SCN}$ & $0.8 \%$ & $0.1 \%$ & $0.2 \%$ & $0.4 \%$ & $94.8 \%$ & $0.0 \%$ & $3.8 \%$ & $100.0 \%$ \\
\hline & 4 & Count & $1128 \mathrm{a}$ & 0a,b,c,d & $21 \mathrm{a}, \mathrm{d}$ & $31 \mathrm{c}$ & 16492b,d & $47 \mathrm{e}$ & $492 b, d$ & 18211 \\
\hline & 4 & $\%$ wT-SCN & $6.2 \%$ & $0.0 \%$ & $0.1 \%$ & $0.2 \%$ & $90.6 \%$ & $0.3 \%$ & $2.7 \%$ & $100.0 \%$ \\
\hline Total & & Count & 3461 & 12 & 59 & 250 & 55851 & 47 & 1770 & 61450 \\
\hline & & $\% \mathrm{wT}-\mathrm{SCN}$ & $5.6 \%$ & $0.0 \%$ & $0.1 \%$ & $0.4 \%$ & $90.9 \%$ & $0.1 \%$ & $2.9 \%$ & $100.0 \%$ \\
\hline
\end{tabular}

Each subscript letter denotes a subset of Terminate Cause categories whose column proportions do not differ significantly from each

Internet Service Delivery Experience (ISDE); (\%wT-SCN); Two-Step Cluster No (T-SCN); Satisfied (S); Very Dissatisfied (VDS); Dissatisfied (DS); Very Satisfied (VS)

\section{3 | Brief Discussion}

For easy discussion, the causes of network termination coded as AcctTerminateCause (see Figure 1) for data analysis are hereafter referred to as Terminate Cause Account (TCA). In Cluster 4, compared to the other three clusters, the TCA showed the highest Lost of Service level (L-S) (see Figure 1). In the same cluster - 4, the number of users' activities totaled $29.6 \%$ of 18 211 users' activities, with that of upload equated to 4,248,016.92. This implied a low data download activity when compared to others. The second-longest session time was 3,753.09, and it was observed in cluster 4. The most frequent Distance was observed in category three and within-cluster 4 indicating $60.5 \%$ of users. The remaining $39.5 \%$ of users within the Distance were observed in category 2 . The users within cluster 4 were mostly spread among ten nodes, and $56.3 \%$ of the users used the wireless network, and the remaining percentage used the fiber optic-based network. The TCA recorded a $90.6 \%$ loss of service due to the Distance the wireless network could not cover. Table 8 supports this claim by showing that L-S was the main cause of Internet service termination in all the clusters. This claim, particularly of the user's influence, is consistent with what obtains in the literature. That is, the closer a user of Internet service is to a service provider's transmitter (in this case, NAS), the better the signal (or service) the user stands to 16 .

The cell distribution presented in Figure 2 represents that of Distance alone as a KPI. Others were omitted for the sake of brevity. The cell distribution reveals that the highest count of users is found in cluster 3 and distance 1 (see the two thick colored bars in Figure 2), which is the closest users' IAN to the NAS's location in the university's ICT center. In the literature, location as a factor has been found to influence the performance of the internet in terms of ISDE to users ${ }^{[4]}$, thus consistent with the findings in this paper. Ultimately, it was observed that users' ISDE could be observed to a sufficient degree to keep users using the Internet service to some extent in any of the five (5) distances. Particularly, it is interesting to observe that when energy is readily available, with the right technology and technical know-how with expert knowledge, deployed users have struggled to cope. All these simply informed the fact that users often migrate to where they can get satisfactory services. The result in Table 3 corroborated this claim. It showed that more than one user was found in the VG category with $28.4 \%$ equaled 17423 users' activities at the user IAN closest to the ICT center. Although it was observed that performance was too poor in terms of QoS, users were there anyway to use the services since the QoS they can get was only available there. Location and QoS are two factors known in the literature to influence each other ${ }^{[29.45]}$, thus highlighting the result presented as plausible.

The scaling applied to get users' responses were two types, namely Very Good (VG), Good (G), Average (AV), Bad (B), and Very Bad (VB); and Very Dissatisfied (VDS), Dissatisfied (DS), Satisfied (S), and Very Satisfied (VS). They were vital in the results reported in Tables 3, 4, 5, 6, and 7. Consistent with the practice in Joumblatt et al. ${ }^{[30}$ and Alismail and Zhang ${ }^{[46]}$, this scaling helped explore participants' experiences in this study. The data analysis results reported in the Tables highlighted earlier helped to understand the interpretations, perceptions, and opinions of users of the university Internet who took part in the study. Based on their perception and insights in the specific university context and as shown in the results presented, it is possible to conclude that a university's Internet performance can be deduced from the services' users. 


\section{5 | CONCLUSION}

The cell distribution presented in Figure 2 represents that of Distance alone as a KPI. Others were omitted for the sake of brevity. The cell distribution reveals that the highest count of users is found in cluster 3 and distance 1 (see the two thick colored bars in Figure 2), which is the closest users' IAN to the NAS's location in the university's ICT center. In the literature, location as a factor has been found to influence the performance of the internet in terms of ISDE to users ${ }^{[4]}$, thus consistent with the findings in this paper. Ultimately, it was observed that users' ISDE could be observed to a sufficient degree to keep users using the Internet service to some extent in any of the five (5) distances. Particularly, it is interesting to observe that when energy is readily available, with the right technology and technical know-how with expert knowledge, deployed users have struggled to cope. All these simply informed This study presented analytic results that show users' activities, which reflect their use of the case study university's Internet services. It also contributed KPIs and the user satisfaction criterion as plausible variables to investigate a university's Internet services' performance based on these results. Answers to two basic questions serve as stepping stones to the conclusions made in this paper. The questions include; (i) is there a convincing connection(s) between KPIs and the level of users' satisfaction with the services offered by the university's Internet service, and (ii) what the possible causes of the loss of Internet service(s) and eventual termination are? In a bid to answer these questions, the theory of user-centricity and the techniques applied came to the limelight as a plausible methodology that is suggested as reliable to user-centrically examine KPIs with user-centric data to study the performance of the Internet services of a university. Based on the results presented in this study, it was found that convincing evidence of relationships and connection between the KPI and user satisfaction exists. This answered the first question and showed the inevitability of using clustering potentials with related techniques to learn patterns of associations that highlight what influences a university's Internet service performance. Adding to this is the knowledge of the factors that causes the eventual termination of a university's Internet services. As the answer to the second question, it revealed the causes of the loss of Internet services. The causes were discovered to be admin reboot, admin reset, lost carrier, loss of service, session timeout, and user request, as shown in Table 8 . The result also showed that loss of service had the highest level of influence concerning the cause of Internet service termination.

Based on this study's context, we conclude that the satisfaction of users with a university's Internet services depends on what users can do with the services provided. Applying KPIs to analyze users' data revealed users' perceptions and opinions concerning the performance of the case study university's Internet services. It highlighted the fact that a university's Internet performance can be deduced from its users. In the future, the QoIS based on QoS, but from the users' perspective, QoE will be addressed user-centrically. This implies the identification and characterization of extensible factors from users' perspectives such that after their use within a university's context, they can be extended to others. Attempt to be more categorical in terms of the Distance between IAN and NAS, unlike what was done in this study, will be given attention. This way, location as Distance would hopefully be better associated with quantitative terms, unlike the qualitative approach employed in this study.

\section{6 | ACKNOWLEDGMENT}

The authors seize this opportunity to appreciate all those - colleagues and family members, and the reviewers to mention but a few - who supported and contributed to this paper's realization. Those who contributed to the research that results in the publication are also acknowledged.

\section{References}

1. Chițescu RI, Frînculeasa MN, Băiaşu D, Chițescu RI, Nicoleta MF, Băiaşu D. Scientific Information and the Internet in the Modern Educational Context. In: 6th BASIQ International Conference on New Trends in Sustainable Business and Consumption. Messina Messina: Bucharest: ASE; 2020. p. 1085-1092. https: //www.researchgate.net/profile/Bassel-Diab/publication/342124082\{_\}BASIQ\{_\}2020\{_\}Conference\{_\}proceedings/ links/5ee37056458515814a583fe1/BASIQ-2020-Conference-proceedings.pdf $\{\#\}$ page=1085

2. Madukoma E, Opeke RO. Information use and job performance of senior nonacademic staff in Nigerian universities. Library Philosophy and Practice 2013;9:1-20. https://digitalcommons.unl.edu/libphilprac/973/ 
3. Reyna J, Hanham J, Meier P. The Internet explosion, digital media principles and implications to communicate effectively in the digital space. E-Learning and Digital Media 2018;15(1):36-52.

4. G A A. Charging for computer networks at higher educational institutions in developing countries. South African Journal of Information and Communication 2008;9:58-67.

5. Austin A, Craig SL, Navega N, McInroy LB. It's my safe space: The life-saving role of the internet in the lives of transgender and gender diverse youth. International Journal of Transgender Health 2020;21(1):33-44.

6. Fitria NA, Yuliati E. The Impact of Behavior of Restaurant Employees on Word of Mouth Intention: The Mediating Role of Customer Satisfaction. IPTEK The Journal for Technology and Science 2020 may;31(1):91-100. https://iptek.its.ac.id/ index.php/jts/article/view/6328

7. Alrwashdeh M, Jahmani A, Ibrahim B, Aljuhmani HY. Data to model the effects of perceived telecommunication service quality and value on the degree of user satisfaction and e-WOM among telecommunications users in North Cyprus. Data in Brief 2020;28:104981.

8. Chen LD, Soliman KS, Mao E, Frolick MN. Measuring user satisfaction with data warehouses: An exploratory study. Information \& management 2000;37(3):103-110.

9. Wu M, Xiao Y, Gao Y, Xiao M. Dynamic Socially-Motivated D2D Relay Selection with Uniform QoE Criterion for Multi-Demands. IEEE Transactions on Communications 2020;68(6):3355-3368.

10. Jaradat A, Alhussian H, Patel A, Fati SM. Multiple users replica selection in data grids for fair user satisfaction: A hybrid approach. Computer Standards and Interfaces 2020;71:1-20.

11. Jiang T, Chi Y, Gao H. A clickstream data analysis of Chinese academic library OPAC users' information behavior. Library and Information Science Research 2017;39(3):213-223.

12. Sieverink F, Kelders S, Poel M, van Gemert-Pijnen L. Opening the Black Box of Electronic Health: Collecting, Analyzing, and Interpreting Log Data. JMIR Research Protocols 2017;6(8):1-12.

13. Creswell JW, Hirose M. Mixed methods and survey research in family medicine and community health. Family Medicine and Community Health 2019;7(2):1-6.

14. Yi-Wen Z, Yan-Qi B, An-Ju Y. Statistical analysis of city and the villages Internet users based on user logs. In: Lu H, Pei X, Su J, editors. MATEC Web of Conferences, vol. 176 Luoyang International Exhibition Center, China; 2018. p. 1-3. https://www.matec-conferences.org/articles/matecconf/abs/2018/35/matecconf \{_\}ifid2018\{_\}03011/ matecconf\{_\}ifid2018\{_\}03011.html

15. Tao Y, Guo S, Shi C, Chu D. User Behavior Analysis by Cross-Domain Log Data Fusion. IEEE Access 2020;8:400-406.

16. Akanni J, Isa AA, Alao RA, Thomas CT. Assessment of Internet Service Provided using UMTS operators at the University of Ilorin main campus. Nigerian Journal of Technology 2020;39(2):500-505.

17. Ferreira A, Antunes P, Pino JA. Evaluating shared workspace performance using human information processing models. Information Research 2009;14(1):388.

18. AKHIGBE B, ADERIBIGBE S, AFOLABI B. Evaluating Web-based Technologies: The Paradigm of User-centricity. Journal of Applied Computer Science \& Mathematics 2016;10(2):32-39.

19. Miller JS, Mondal A, Potharaju R, Dinda PA, Kuzmanovic A. Understanding end-user perception of network problems. In: Proceedings of the 1st ACM SIGCOMM Workshop on Measurements Up the Stack, W-MUST'11 Toronto Ontario, Canada: ACM SIGCOMM; 2011. p. 43-48. https://dl.acm.org/doi/10.1145/2018602.2018613.

20. Doll WJ, Torkzadeh G. The Measurement of End-User Computing Satisfaction: Theoretical and Methodological Issues. MIS Quarterly 1991;15(1):5-10. 
21. Patomäki H, Wight C. After postpositivism? The promises of critical realism. International Studies Quarterly 2000;44(2):213-237.

22. Haryadi S. Performance Measurement of Internet Service. Jakarta; 2018.

23. Huston G. Measuring IP Network Performance A Functional Definition of Network Performance. The Internet Protocol Journal 2003 mar;6(1):2-11.

24. Cherkasova L, Tang W, Fu Y, Vahdat A. Measuring and Characterizing End-to-End Internet Service Performance. ACM Transactions on Internet Technology 2003;3(4):347-391.

25. Clark M. 4. In: Clark M, editor. Quality of Service (QOS), Network Performance and Optimisation New York, USA: John Wiley \& Sons, Ltd; 2003. p. 565-610.

26. Nagaraja K. A systematic approach to quantifying and improving the availability of internet services I Guide books. PhD thesis, Rutgers University; 2006.

27. Borzemski L. Data Mining in Evaluation of Internet Path Performance. In: Orchard B, Yang C, Ali M, editors. Innovations in Applied Artificial Intelligence Berlin, Heidelberg: Springer Berlin Heidelberg; 2004. p. 643-652.

28. Polpinij J, Namee K. Internet usage patterns mining from firewall event logs. In: ACM International Conference Proceeding Series London, UK: Association for Computing Machinery; 2019. p. 93-97. https://dl.acm.org/doi/proceedings/10.1145/ 3322134

29. Liston JR. Measuring User-Perceived Internet Performance in Multiple Locations. PhD thesis, Georgia Institute of Technology; 2004.

30. Joumblatt D, Chandrashekar J, Kveton B, Taft N, Teixeira R. Predicting user dissatisfaction with Internet application performance at end-hosts. In: Proceedings - IEEE INFOCOM; 2013. p. 235-239.

31. Shaikh J, Fiedler M, Collange D, Arlos P, Minhas TN. Inferring User-perceived Performance of Network by monitoring TCP Interruptions. Network Protocols and Algorithms 2012;4(2):49-67.

32. Lin HY, Hsieh SH, Chen KH, Wu TC. Large-scale mobile internet performance measurement in Taiwan. In: International Conference on Ubiquitous and Future Networks, ICUFN Shanghai, China: IEEE Comput. Soc; 2014. p. 516-519.

33. Chen TM, Hu L. Internet performance monitoring. Proceedings of the IEEE 2002;90(9):1592-1603.

34. Bajpai V, Schonwalder J. A survey on internet performance measurement platforms and related standardization efforts. IEEE Communications Surveys and Tutorials 2015;17(3):1313-1341.

35. Ani OE, Edem MB, Ottong EJ. Analysis of internet access and use by academic staff in the University of Calabar, Calabar, Nigeria. Library Management 2010;31(7):535-545.

36. Ehikhamenor FA. Internet facilities: use and non-use by Nigerian university scientists. Journal of Information Science 2003;29(1):35-48. https://doi.org/10.1177/016555150302900104.

37. Shihm MY, Jheng JW, Lai LF. A two-step method for clustering mixed categroical and numeric data. Tamkang Journal of Science and Engineering 2010;13(1):11-22.

38. Tang D, Dai R, Tang L, Li X. Low-rate DoS attack detection based on two-step cluster analysis and UTR analysis. Humancentric Computing and Information Sciences 2020;10(1):1-20.

39. Şchiopu D. Applying TwoStep Cluster Analysis for Identifying Bank Customers' Profile. BULETINUL 2010;62(3):66-75. http://support.spss.com/productsext/spss/documentation/.

40. Ledger S, Ersozlu Z, Fischetti J. Preservice teachers' confidence and preferred teaching strategies using teachlive ${ }^{\mathrm{TM}}$ virtual learning environment: A two-step cluster analysis. Eurasia Journal of Mathematics, Science and Technology Education 2019;15(3):1-17. 
41. Wendler T, Gröttrup S. Data mining with SPSS modeler: Theory, exercises and solutions. Berlin: Springer International Publishing; 2016.

42. Berba EM, Palaoag TD. Improving customer satisfaction on internet services in LNU using virtualized AAA network architecture. In: ACM International Conference Proceeding Series Osaka, Japan: Association for Computing Machinery (ACM); 2019. p. 178-183. https://dl.acm.org/doi/10.1145/3323716.3323729

43. Munyanti I, Masrom M. Customer Satisfaction Factors towards Mobile Network Services. Journal of Advanced Research in Business and Management Studies 2018;13(1):9-18. http://www.akademiabaru.com/doc/ARBMSV13\{_\}N1\{_\}P9\{_\}18. pdf

44. Ghose A, Han SP. An empirical analysis of user content generation and usage behavior on the mobile Internet. Management Science 2011;57(9):1671-1691.

45. Wang Q, Wang X, Ding X. QoS Attributes Prediction with Attention-based LSTM Network for Mobile Services. In: 5th International Conference on Big Data Computing and Communications, BIGCOM 2019 QingDao, China: IEEE; 2019. p. 7-11. https://ieeexplore.ieee.org/document/8904992.

46. Alismail S, Zhang H. Exploring and Understanding Participants' Perceptions of Facial Emoji Likert Scales in Online Surveys. ACM Transactions on Social Computing 2020 may;3(2):1-12.

How to cite this article: Ojebode M., Akhigbe B.I. (2020), Integrated Risk Assessment on Argon Purification Unit Based on FMECA and Fuzzy-AHP, IPTEK The Journal of Technology and Science, 31(3):279-294. 